\title{
Sentido y significado de las ocupaciones de las mujeres del Cabildo Indígena de Suba. Perspectivas desde Terapia Ocupacional ${ }^{1}$
}

\author{
Sense and meaning of the occupations of women from the \\ Indigenous Council of Suba. Insights from the Occupational \\ Therapy
}

\author{
Johana Mogollón Cárdenas² \\ Recibido: 27 de enero 2016 • Enviado para modificación: 12 de julio de 2016 • Aceptado: 25 de julio de 2016 \\ Mogollón, J. (2016). Sentido y significado de las ocupaciones de las mujeres del Cabildo Indígena de Suba. Perspectivas desde \\ Terapia Ocupacional. Revista Ocupación Humana, 16 (1), 32-43.
}

\begin{abstract}
Resumen: Esta investigación busca enriquecer la comprensión de los procesos de construcción de sentidos y significados de las ocupaciones en un grupo de mujeres del Cabildo Indígena Muisca de Suba. Este cabildo ofreció la oportunidad de evidenciar dicha construcción, teniendo en cuenta la influencia de las diferentes dinámicas que ha vivido. Se realizaron entrevistas semiestructuradas a nueve mujeres del cabildo con el fin de establecer las transformaciones en la asignación de sentido y significado a las ocupaciones que realizaron en la infancia, la adultez y a las que realizan actualmente; buscando además comprender la influencia de variables contextuales, el género y la vinculación al cabildo en tal asignación. Se evidenció que las ocupaciones de las mujeres que tienen apellidos muiscas (cabildantes) tienen mayor sentido que significado, mientras que las de quienes están casadas con un hombre cabildante (adoptadas) tienen mayor significado. La participación se convierte en una ocupación valorada a través de la cual las mujeres se empoderan dentro de la comunidad indígena. Las variables contextuales influyen en la asignación de sentido y significado en ambos grupos, aunque de manera particular.
\end{abstract}

Palabras clave: población indígena, participación de la mujer, cambio cultural, ocupaciones.

Abstract: This research seeks to enhance the understanding of the processes through which a group of women from the Indigenous Council of Suba construct the senses and meanings of their occupations. This council offered us the opportunity to evidence such construction, taking into account the influence of the different dynamics lived within it. We conducted semi structured interviews with nine women of the council, in order to establish how providing meaning and significance to the occupations performed in childhood, adulthood and to those currently performed has changed; seeking to also understand how the contextual variables, gender and the women's links to the council influence this provision of meaning. It was evident that the occupations of women who have Muisca surnames (lobbyists) have greater

\footnotetext{
${ }^{1}$ Artículo derivado de la investigación Sentido y significado de las ocupaciones de las mujeres del Cabildo Indígena de Suba. Perspectivas desde Terapia Ocupacional, elaborada por la autora como trabajo de grado para optar al título de terapeuta ocupacional de la Universidad Nacional de Colombia y dirigida por la profesora Jeannette Méndez Montaño.

${ }^{2}$ Terapeuta Ocupacional. Especialista en Higiene y Salud Ocupacional. Asesora en seguridad y salud en el Trabajo, As Ocupacional. Bogotá, Colombia. johanamogollonc@gmail.com
} 
sense, whereas those of the ones who are married to lobbyist men (adopted) have greater meaning. Participation becomes a valued occupation through which women are empowered within the indigenous community. The contextual variables influence the provision of sense and meaning in both groups, but in specific ways.

Key words: indigenous populations, women's participation, cultural change, occupations.

\section{Introducción}

Este trabajo de investigación partió del deseo y la necesidad de enriquecer la comprensión sobre las ocupaciones y el sentido y significado atribuido a ellas. Este es un proceso complejo, debido a la consideración que debe hacerse de diversas variables que influyen en su construcción, como el contexto, la cultura, la historia, las dinámicas individuales y sociales, la participación, el género, entre otras (Becerra, Cerquera \& Martínez, 2009; Chindoy \& Puenayán, 2009; Beagan \& D'Sylva, 2011; Galeano, Monsalve \& Wilches, 2012; Méndez, Bravo, Camelo \& Zúñiga, 2013).

El escenario en el cual se llevó a cabo este estudio fue el Cabildo Indígena Muisca de Suba, el más antiguo de los cinco cabildos muiscas reconocidos por el Estado colombiano3. Para llevar a cabo la investigación se establecieron cinco categorías de análisis: ocupación, sentido, significado, cabildo y género; en tres etapas del ciclo vital: infancia, adultez, actualidad (cabildo). Ello con el ánimo de identificar los principales cambios ocupacionales que tuvieron las mujeres, sus posibles causas, así como las transformaciones que se presentaron para asignar sentidos y significados.

Trujillo, Sanabria, Carrizosa y Parra (2011) definen ocupación ${ }^{4}$ como el proceso "a través del cual el ser humano puede desarrollar sus potenciales biológicos, individuales y socioculturales para hacerse sujeto, gestor y constructor de su propia historia" (p. 50); esta "aporta significación a la existencia humana, y los seres humanos adjudican significados individuales y colectivos a sus ocupaciones" (p. 56).

Así mismo, Zemke (2004) y Rowles (2008) indican que la ocupación depende del tiempo y del espacio. Wilcock (citada por Navarrete, 2010) considera que "las ocupaciones son procesos dinámicos en los que interviene la cultura a la que pertenece una persona" (p. 137). En tanto Townsend (citada por Trujillo et al., 2011) se refiere

\footnotetext{
${ }^{3}$ Un cabildo es "la reunión de familias que tienen su territorio, permanecen en él conservando sus culturas y tradiciones" (fragmento de la entrevista realizada a Luis Yopasá, Alcalde Mayor del Cabildo Muisca de Suba, 2013). En Colombia, según el Decreto 2164 de 1995, un cabildo es "una entidad pública especial cuyos integrantes son miembros de una comunidad indígena, elegidos y reconocidos por esta, con una organización sociopolítica tradicional, cuya función es representar legalmente a la comunidad, ejercer la autoridad y realizar las actividades que les atribuyan las leyes, los usos, las costumbres y el reglamento interno de cada comunidad". ${ }^{4}$ Tres de las principales categorías analizadas en la investigación son: ocupación, sentido y significado. La autora hizo una revisión de fuentes nacionales e internacionales que las abordan, esta puede ser consultada en el informe completo, disponible en: http://www.bdigital.unal.edu.co/11403/1/581236.2013.pdf
} 
a la ocupación como un "proceso activo de vivir" (p. 45), que posibilita la transformación personal, las acciones interpersonales y los procesos emancipatorios de equidad y justicia.

Argüelles y Pérez (citados por Méndez et al., 2013) consideran que el sentido es el valor personal asignado a las ocupaciones, se define por el por qué y para qué se realizan, junto con la importancia y el papel que desempeñan en la vida de las personas. Involucra los aportes que cada ocupación le brinda a un sujeto inmerso en un contexto con características particulares.

Para los efectos de este estudio se considera que el sentido se relaciona con las razones por las cuales se elige o realiza una ocupación; los sentimientos de disfrute, alegría / felicidad y satisfacción que genera; las posibilidades que brinda, la oportunidad de conectar el pasado, el presente y el futuro; la posibilidad de compartir con otros, la instrumentalidad de la ocupación o su utilidad para realizar proyectos de vida; los sentimientos de productividad, satisfacción de necesidades personales y la evocación del hogar y los ancestros que se logran a partir del desempeño de ocupaciones; también depende del tiempo y del espacio, y de factores contextuales, sociales y culturales (Persson, Erlandsson, Eklund, \& Iwarsson, 2001; Bundgaard, 2005; Ikiugu, 2005; Leufstadius, Erlandsson, Björkman y Eklund (2008); Reed, Hocking \& Smythe, 2010; Beagan \& D'Sylva, 2011). Cabe resaltar además que el contexto del cual proviene un individuo y los cambios que tienen lugar en su vida impactan en la atribución de valor y sentido para la ocupación (Christiansen \& Baum, citados por Yazdani, 2012).

Por otro lado, el significado es el valor social que se asigna a las ocupaciones que una persona realiza (Bravo, Camelo \& Zúñiga, 2011; Galeano et al., 2012). Cutchin, Aldrich, Bailliard y Coppola (2008) agregan que la adscripción de significados es un proceso subjetivo, o sea que ocurre en el fuero interno de la persona a partir de sus percepciones y experiencias. Para mayor claridad, en esta investigación se analizó el significado desde la consideración y percepción que tienen las mujeres sobre cómo el colectivo valora las actividades y el papel que ellas han desempeñado dentro y fuera del cabildo; se relaciona directamente con el poco o mucho reconocimiento que reciben por lo que hacen y los sentimientos que tal reconocimiento les genera.

En cuanto a la categoría género, se buscó analizar y comprender las oportunidades que las mujeres han tenido para elegir y participar en ocupaciones durante toda su vida, además de visibilizar los mecanismos por los cuales se definen las cosas que debían y deben hacer hombres y mujeres dentro de sus contextos, y cómo ha sido su participación en las actividades que se llevan a cabo dentro del Cabildo.

Finalmente, el Cabildo fue analizado desde las concepciones y consideraciones que las mujeres percibían del funcionamiento de este como comunidad. Incluye el reconocimiento que dan a las dinámicas propuestas en él para mantener y recuperar una identidad indígena Muisca, junto con el establecimiento de relaciones inter- 
personales, la identificación de territorios propios y el conocimiento de los procesos de ruptura que ha sufrido su comunidad a lo largo de la historia.

Las dinámicas de disgregación, las rupturas históricas provocadas por la colonización española y la urbanización de Suba -a raíz del cambio de su figura como municipio a localidad de la ciudad de Bogotá- causaron que el espectro de ocupaciones que realizaban los descendientes de los indígenas se modificara. Durante el desarrollo de la investigación se indagó sobre la cultura Muisca a lo largo de sus principales momentos históricos, teniendo en cuenta no solo fuentes documentales, sino también los testimonios de historiadores y otros miembros del Cabildo ${ }^{5}$.

En tiempos de la Conquista, la comunidad indígena Muisca se vio obligada a relegar sus costumbres, tradiciones, prácticas religiosas y espirituales, y en general todas sus formas de vida, a causa de la dominación española. Los muiscas tuvieron que adoptar una religión monoteísta -la católica- junto con hábitos y ocupaciones totalmente diferentes a los que practicaban desde sus inicios. Ese momento histórico representó una ruptura y una disgregación de esta comunidad, que luchó por defender y mantener sus costumbres; quien se opusiera a los designios de los españoles debía afrontar serios castigos, incluso la muerte, razón por la cual muchos muiscas se refugiaron en diferentes sectores del altiplano cundiboyacense, principalmente en
Suba, Bosa, Cota, Chía y Sesquilé. Dicho proceso provocó que se comenzaran a perder y transformar sus tradiciones y prácticas (Londoño, 1988).

A través de las entrevistas realizadas a miembros de la comunidad se pudo conocer que hacia 1988 deciden reorganizarse y recuperar sus terrenos y su identidad como muiscas, por esta razón se conforma el Cabildo Indígena de Suba. En este contexto, las personas que tengan algún apellido reconocido por el Cabildo como Muisca tienen el derecho de vincularse a él. Tal vinculación representa una serie de ayudas y subvenciones que incluyen acceso a la canasta familiar, beneficios en salud y educación, libreta militar, becas universitarias y beneficios para el ingreso a la educación superior, desayunos para niños, actividades deportivas y recreativas.

Con el fin de recuperar todas aquellas costumbres perdidas, el Cabildo ha sumado esfuerzos para proponer actividades que realizaban los muiscas y que construían su identidad: tejido, ollas comunitarias, club de la palabra, consejo de niños, de jóvenes, de mujeres y de abuelos, entre otras. Sin embargo, algunos líderes del Cabildo reconocen y consideran que si bien hay descendientes de los muiscas que participan en dichas actividades porque quieren recuperar su identidad como indígenas, existen otros que participan exclusivamente por la necesidad de acceder a los beneficios derivados de pertenecer a una comunidad indígena reconocida a nivel distrital y nacional.

\footnotetext{
${ }^{5}$ Se invita al lector a revisar el informe de investigación para ampliar los hallazgos relacionados con la historia del Cabildo y las transformaciones históricas, culturales, económicas y geográficas que ha vivido la comunidad Muisca.
} 
Con lo anterior se evidencia que la comunidad Muisca ha vivido diferentes situaciones en su historia, por lo cual el espectro de ocupaciones y la construcción de sentidos y significados asociados a ellas se ha visto influenciado por factores culturales, políticos, sociales, espaciales y temporales.

Otra consecuencia de ese transcurrir es el cambio en la consideración de la mujer. De acuerdo a los relatos de las personas entrevistadas, en el pasado regía una organización social en la cual imperaba el matriarcado ${ }^{6}$, no obstante, la mayoría de las decisiones y las posibilidades de participación de las mujeres dependían de las exigencias del contexto y de los designios de sus familiares. La distribución de roles, así como las posibilidades de elección de las ocupaciones también se han modificado con el tiempo.

A partir de las anteriores consideraciones, esta investigación pretende identificar las transformaciones que se han dado en la construcción de sentidos y significados de las ocupaciones de las mujeres del Cabildo Indígena de Suba, teniendo en cuenta la influencia de factores históricos, culturales, económicos y geográficos.

\section{Metodología}

Se realizó un estudio de tipo cualitativo - descriptivo ${ }^{7}$, en el cual se efectuaron entrevistas semiestructuradas a nueve mujeres pertenecientes al Ca- bildo. Seis de ellas vinculadas por ser descendientes directas de los Muiscas, debido a que poseen algún apellido de los clanes (en el cabildo se les llama cabildantes). Las otras tres, vinculadas por estar casadas con un hombre de apellido reconocido como Muisca, a quienes se les denomina adoptadas.

Las categorías analizadas fueron: ocupación, sentido, significado, cabildo y género (términos ampliados en la introducción). Con el fin de analizarlas se diseñó una entrevista con 36 preguntas, por medio de las cuales se buscó reconocer: las ocupaciones que han desempeñado las mujeres cabildantes y adoptadas de la comunidad, desde que eran niñas hasta la actualidad; los sentimientos, aportes e importancia que dichas ocupaciones han tenido para ellas (sentido), y la importancia y valoración que otras personas han dado a dichas ocupaciones y su participación en el Cabildo (significado).

En cuanto al género, se plantearon preguntas relacionadas con las posibilidades que las participantes tuvieron en diferentes momentos de su ciclo vital para desempeñar ocupaciones, y si han tenido limitaciones para elegirlas libremente por el hecho de ser mujeres. También se incluyeron preguntas concernientes a la percepción de interés y participación en las dinámicas del Cabildo, teniendo en cuenta ambos géneros.

\footnotetext{
${ }^{6}$ Término utilizado por las mujeres entrevistadas para hacer referencia a la estructura familiar y social en la cual la mujer tenía una posición de poder (más social que político), ya que era vista como un pilar fundamental de la misma.

${ }^{7}$ Acevedo y García (2012) argumentan que la investigación cualitativa es de gran utilidad para los estudios de Terapia Ocupacional con comunidades indígenas, ya que la información obtenida de la comunidad es contada por sus propios miembros, describiendo aspectos importantes y de interés.
} 
Con respecto al Cabildo, se establecieron preguntas encaminadas a indagar sobre el agrado o desagrado que experimentan las mujeres por participar en las actividades del mismo; los aportes, objetivos e intereses que persiguen al momento de comprometerse en las dinámicas propuestas, y las actividades que les gustaría proponer.

Antes de las entrevistas, todas las mujeres participantes conocieron los objetivos del estudio, aceptaron participar voluntariamente y autorizaron la grabación de las mismas.

El análisis de las entrevistas consistió en una clasificación de ocupaciones por etapas (infancia, adolescencia conformación de familias y vida adulta - vinculación al Cabildo); la identificación de categorías de asignación de sentido a las ocupaciones desempeñadas en las diferentes etapas (acordes con las señaladas por diferentes autores); así como de los procesos de asig- nación de significado. También se hizo el respectivo análisis de las preguntas relacionadas con las categorías género y Cabildo.

\section{Resultados}

\section{Ocupación}

Infancia. Las mujeres cabildantes y las adoptadas desempeñaban ocupaciones similares, teniendo en cuenta que la mayoría nació y creció en el campo. Se identificaron diversas categorías de ocupación para cada grupo (Tabla 1); las mujeres adoptadas manifestaron no realizar actividades de autocuidado y religiosas cuando eran niñas, argumentando falta de tiempo por la necesidad de encargarse de los oficios del hogar. Otro punto importante de contraste se encuentra en el juego; las cabildantes refirieron que tenían mucho tiempo y personas a su alrededor para jugar, elementos que resultaban escasos para las adoptadas.

Tabla 1. Ocupaciones de las mujeres en la infancia.

\begin{tabular}{|l|l|}
\multicolumn{1}{|c|}{ Mujeres cabildantes } & \multicolumn{1}{c|}{ Mujeres adoptadas } \\
\hline Actividades agrícolas - productivas & Actividades agrícolas - productivas \\
\hline Cuidado del hogar & Cuidado del hogar \\
\hline Estudio & Estudio \\
\hline Juego - esparcimiento & Juego - esparcimiento \\
\hline Autocuidado & \\
\hline Tradición oral, unión familiar, costumbres ancestrales & Unión familiar \\
\hline Religiosas / espirituales & \\
\hline
\end{tabular}

Fuente: Elaboración propia.

Los cambios ocupacionales que las mujeres vivieron se debieron a causas similares en ambos grupos: mientras las cabildantes tuvieron que cambiar sus ocupaciones a causa de la urba- nización de Suba, la conformación de sus familias y la vinculación al Cabildo, las adoptadas tuvieron que hacerlo por el desplazamiento desde sectores rurales a urbanos, por la conformación 
de su familias y la vinculación al Cabildo.

Vida adulta y actualidad. Teniendo en cuenta las razones por las cuales las mujeres de ambos grupos tuvieron que modificar el espectro de sus ocupaciones, se evidenció que las adoptadas y las cabildantes dejaron de realizar tareas agrícolas para comenzar a desempeñar otro tipo de actividades laborales con el fin de mantener a sus familias. En cuanto al juego, si bien no se abandonó, si se modificó, ya que empezaron a jugar con sus propios hijos.

Actualmente las nueve mujeres participan en las actividades propuestas por el Cabildo, realizan tejidos, asisten a reuniones y a festividades especiales para los muiscas. Es importante resaltar que ambos grupos manifestaron que la participación es una ocupación importante, a través de la cual pueden emprender procesos de empoderamiento. Las cabildantes se reconocen como lideresas y protagonistas en la recuperación de su cultura, en la consolidación de su comunidad y en el fortalecimiento de su identidad como mujeres y como descendientes de los muiscas. Para las adoptadas, la posibilidad que tienen de aprender nuevas cosas y mejorar sus habilidades se convierte en un medio para sentirse mejor con ellas mismas y hacer parte de una comunidad que las reconoce y las acoge como miembros fundamentales.

\section{Sentido}

Infancia. Tanto las mujeres cabildantes como las adoptadas refieren que se sintieron bien realizando sus ocupaciones de niñas; sin embargo, las cabildantes fueron más enfáticas en reconocer que se sentían felices (Ikiugu, 2005; Leufstadius et al., 2008), no solo por el lugar donde vivían y realizaban sus actividades, sino también por las personas con quienes podían hacerlo (Reed, et al., 2010) y por la posibilidad de aprender sobre su cultura y sus raíces ancestrales (Beagan \& D'Sylva, 2011). Por otro lado, las mujeres adoptadas atribuyeron importancia a sus ocupaciones de la infancia por la satisfacción de necesidades personales (Bundgaard, 2005) y por convertirse en un medio para aprender a desempeñar las labores del hogar para cuando conformaran los propios (instrumentalidad de las ocupaciones ${ }^{8}$ : Ikiugu, 2005).

Vida adulta. El sentido atribuido a las ocupaciones en esta etapa de sus vidas se relaciona principalmente con la satisfacción de necesidades personales y familiares (Bundgaard, 2005; Reed et al., 2010) y el fortalecimiento de sus proyectos de vida (Ikiugu, 2005).

Actualidad - Cabildo. En esta etapa se encuentran las mayores diferencias entre grupos. Las cabildantes consideran que las actividades realizadas en el Cabildo les permiten conectarse con el pasado, recordar sus ocupaciones más valoradas -las de la infancia- y recuperar sus costumbres ancestrales (Reed et al., 2010). Las adoptadas consideran importantes las actividades que realizan en el Cabildo debido a que pueden compartir con otras personas y aprender a hacer diferentes cosas

\footnotetext{
${ }^{8}$ Utilidad de la ocupación para realizar proyectos de vida.
} 
que más adelante pueden vender, razón por la cual se sienten productivas y realizadas (instrumentalidad de las ocupaciones: Ikiugu, 2005; valor tangible y simbólico de las ocupaciones: Persson, et al., 2001) .

\section{Significado}

Para esta categoría también se evidencian cambios importantes según las etapas del ciclo vital en ambos grupos de mujeres.

Infancia. Mientras las mujeres cabildantes recibían un alto reconocimiento por sus ocupaciones, para las adoptadas el reconocimiento y agradecimiento eran menores.

Vida adulta. En ambos grupos se evidenció un bajo reconocimiento por el desempeño de sus ocupaciones, relacionadas con el mantenimiento y cuidado del hogar, trabajo y estudio.

Actualidad - Cabildo. Aunque las mujeres cabildantes lideran proyectos para su comunidad, reciben un bajo reconocimiento por sus ocupaciones y sus esfuerzos; en contraste, las mujeres adoptadas reciben un alto reconocimiento por vincularse y participar en las actividades propuestas en el Cabildo, ya que sus más allegados consideran que estas se convierten en un medio importante para ocupar el tiempo libre, aprender nuevas cosas y obtener ganancias.

\section{Cabildo}

Las mujeres consideran al Cabildo como un espacio, un lugar en el cual pueden asumir un papel protagónico por medio del cual se empoderan de los procesos que suceden en la comunidad.
Así mismo, el Cabildo es reconocido como un espacio alegre, lleno de vida e historia, en el cual se pueden desempeñar y recuperar aquellas ocupaciones que más valoran.

\section{Género}

Teniendo en cuenta las posibilidades de participación de las mujeres en diferentes ocupaciones a lo largo de su vida, se evidenció que para las cabildantes se tenían más en cuenta las características biológicas para establecer lo que podían hacer hombres o mujeres, es decir, no había condiciones o designios culturales para la división de ocupaciones de acuerdo al género.

Por el contrario, las mujeres adoptadas refirieron que el desempeño de sus actividades se veía limitado, esto debido a la división de ocupaciones entre géneros por el predominio de un modelo machista, en el cual los hombres salían a trabajar y las mujeres debían quedarse en casa.

Para las mujeres de ambos grupos, en la actualidad existe mayor equidad para que hombres y mujeres realicen las mismas actividades.

\section{Discusión}

Teniendo en cuenta el desempeño de ocupaciones de las mujeres de ambos grupos en las diferentes etapas del ciclo vital analizadas, se logra observar lo expuesto por Rowles (2008) y Zemke (2004), en cuanto a que la elección y realización de actividades, así como la asignación de sentido y significado, dependen del lugar y el momento en donde pudieron o pueden ser ejecutadas. Así por ejemplo, las ocupaciones más valoradas por las cabildantes 
son aquellas que realizaron en la infancia, ya que vivían en las casas de sus abuelos y podían compartir con sus familias.

Del mismo modo, las ocupaciones realizadas por las mujeres cabildantes y adoptadas a lo largo de su vida les han permitido desarrollar sus potenciales biológicos, individuales y socioculturales (Rojas, 2011), estos últimos, principalmente en las cabildantes.

En la infancia los potenciales individuales de las mujeres se veían favorecidos a través del establecimiento de buenas relaciones con sus familiares (en el caso de las mujeres adoptadas, las labores del campo y del hogar eran realizadas con los familiares más cercanos -padres y hermanos(as)-, mientras que las mujeres cabildantes compartían con toda su familia, la cual vivía en un mismo espacio) y por el crecimiento personal que brindaba la realización de actividades agrícolas, el cuidado del hogar, el cuidado de animales, etc., en cuanto les daban "bases para una vida adulta" y aprendizajes para "ganarse la vida".

Así mismo, se confirma lo expuesto por Bravo et al. (2011) y Méndez et al. (2013) respecto a la participación como ocupación que desarrolla el potencial individual, en tanto favorece un proceso de empoderamiento a través de la construcción y el fortalecimiento de su identidad como mujeres, con la capacidad de liderar la recuperación de las tradiciones, usos y costumbres de sus ancestros muiscas (en el caso de las cabildantes); de gestionar proyectos, aprender, elegir y participar en actividades cargadas con sentido y significado. Además, la participación en el Cabildo les permite tener momentos de esparcimiento en los cuales pueden compartir experiencias y conocimientos, apoyar la realización de actividades y construir vínculos de amistad (mujeres adoptadas).

En cuanto a la intervención en actividades dentro del Cabildo, se evidencia un proceso de recuperación del legado cultural por medio de las ocupaciones (rituales, tejidos, medicina ancestral, preparación de alimentos), lo que provoca que su participación en el Cabildo se dote de significado. Siguiendo a Townsend (1997), tales ocupaciones posibilitan una transformación personal de las mujeres, en cuanto promueven su empoderamiento por ser lideresas en la comunidad, y una transformación social del Cabildo, gracias a que ellas son protagonistas en un proceso de recuperación de una cultura, aunque sean poco valoradas por los demás.

Sobre la categoría género, se revelan diferencias y cambios relacionados con la elección y la participación en ocupaciones de acuerdo al grupo al que pertenecen las mujeres (cabildantes o adoptadas) y a las etapas del ciclo vital. Las adoptadas tuvieron mayores dificultades en su infancia para elegir libremente qué hacer; el contexto, sobre todo el familiar, disponía lo que hacían hombres o mujeres, limitando a estas a permanecer en casa y a hacer actividades del hogar. Ambos grupos concuerdan en que en la actualidad tienen mayores posibilidades de elegir sus ocupaciones.

Hablando de la participación en ocupaciones dentro del Cabildo, se observa que las mujeres son quienes más se 
han interesado en proponer y participar en los talleres, asambleas y actividades que buscan recuperar y fortalecer la identidad indígena; sin embargo, ellas no reconocen algún motivo que explique dicha diferencia. En este sentido, uno de los límites del estudio es que no se pudieron establecer diferencias en la asignación de sentido según el género, ya que no se incluyeron hombres en la investigación.

Por otro lado, se evidenció que la ocupación, el sentido y el significado son influenciados de manera transversal por variables culturales (identidad, usos y costumbres muiscas; estilo de vida campesino), socio-económicas (urbanización de Suba por necesidades de la ciudad de Bogotá, conformación de las familias, ingreso al campo laboral), socio-históricas (procesos de ruptura y disgregación como consecuencia de la colonización española; cambios en el espectro y desempeño de ocupaciones a causa de la urbanización de Suba; pérdida de costumbres ancestrales y ocupaciones valoradas por las mujeres cabildantes; desplazamiento de las mujeres adoptadas hacia la ciudad) y, finalmente, por variables geográficas (pérdida de los territorios ancestrales, modificaciones de las casonas de los abuelos de las cabildantes; establecimiento de la vivienda y el trabajo en casas del contexto urbano).

Teniendo en cuenta los principales hallazgos del estudio, resulta claro que la construcción de sentidos y significados de las ocupaciones de las mujeres se ha modificado con el tiempo.

Las variables geográficas se relacionan con el espacio, el cual facilita o dificul- ta la realización de ocupaciones y determina cuáles se pueden o deben hacer. También con la afinidad emocional que se ha tenido con los lugares; esta tiene que ver con el sentido que se asigna a las ocupaciones realizadas en tales espacios (Rowles, 2008). Estas dinámicas se ilustran en la Figura 1.

Figura 1. Influencia e interacción de las variables y los factores contextuales.

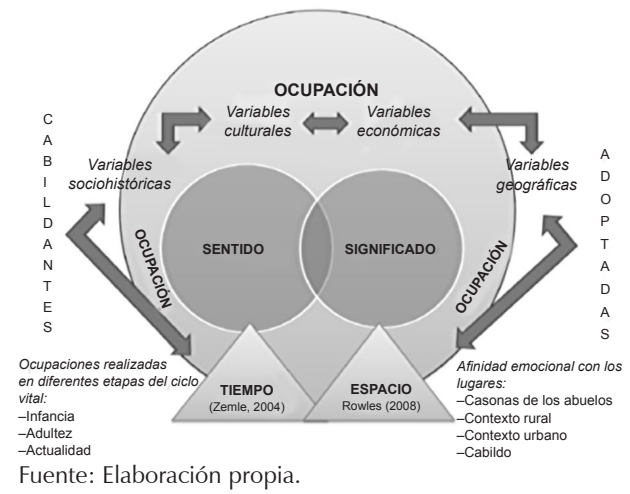

Así mismo, se evidencia que la relación entre sentido y significado varía entre los grupos de mujeres. Esto se resume en la Figura 2.

Figura 2. Interacción del sentido y el significado en ambos grupos de mujeres.

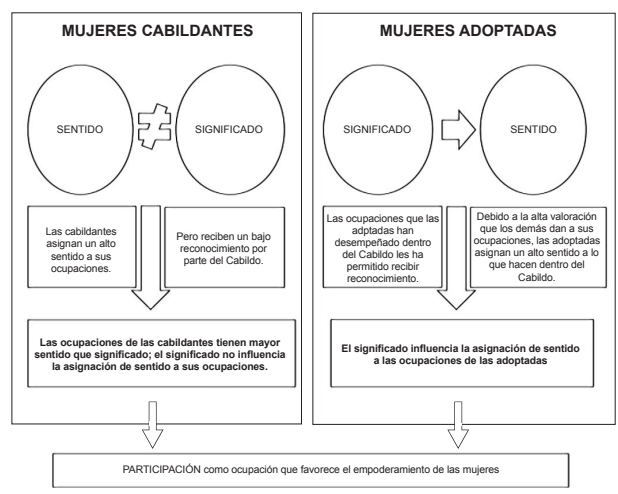

Fuente: Elaboración propia. 


\section{Conclusiones}

Las ocupaciones desempeñadas por las mujeres dentro del Cabildo posibilitan la transformación personal y social, en cuanto favorecen sus potenciales individuales y socioculturales, así como los procesos de recuperación de su identidad cultural.

La participación se convierte en una de las ocupaciones más importantes para las mujeres cabildantes, ya que han emprendido un proceso de empoderamiento en el cual se reconocen como lideresas y protagonistas de la recuperación de su cultura, la consolidación de su comunidad y el fortalecimiento de su identidad como mujeres y como descendientes de los muiscas.

Para las mujeres adoptadas la participación también desempeña un papel importante, ya que han logrado obtener el reconocimiento que no recibieron anteriormente por la realización de sus actividades y están fortaleciendo su identidad como mujeres con capacidad de pertenecer a una comunidad y a la sociedad en general, promoviendo cambios y apoyando, directa o indirectamente, la recuperación de una cultura casi perdida, la de sus esposos con ascendencia indígena.

Se evidencia la influencia del tiempo y el espacio en la asignación de sentido a las ocupaciones de las mujeres cabildantes, ya que este cambió en las diferentes etapas del ciclo vital analizadas. Las ocupaciones cargadas con mayor sentido para ellas son las de la infancia; también se atribuye un alto sentido a las desempeñadas en el Cabildo, ya que les permiten rememorar lo que hacían en sus casas cuando eran ni- ñas, junto a sus abuelos, y recuperar su identidad.

Los hallazgos de esta investigación confirman la importancia de que los terapeutas ocupacionales comprendan la ocupación desde otras perspectivas y consideren los sentidos y significados que las personas atribuyen a lo que hacen al momento de proponer y realizar intervenciones, para así promover la salud y el bienestar a través de actividades valoradas e importantes, que desarrollen los potenciales individuales y socioculturales de los usuarios.

Teniendo en cuenta los aportes de este estudio a la comprensión de los conceptos de ocupación, sentido y significado, se invita a los terapeutas ocupacionales a implementarlos y continuar analizándolos en su hacer para así fortalecer y unificar nuestro conocimiento como profesión.

\section{Referencias}

Acevedo, L., \& García, A. (2012). Haciendo Terapia Ocupacional en Comunidades Indígenas (Tesis de pregrado). Universidad Nacional de Colombia, Bogotá.

Beagan, B., \& D'sylva, A. (2011). Occupational Meanings of Food Preparation for Goan Canadian Women. Journal of Occupational Science, 18 (3), 210 - 222.

Becerra A., Cerquera M., \& Martínez M. (2009). Sentido que le otorgan los hombres y las mujeres al cuidado como ocupación desde sus representaciones culturales (Tesis de pregrado). Universidad Nacional de Colombia, Bogotá.

Bravo C., Camelo N., \& Zuñiga D. (2011). Construcciones de sentido y significado de las ocupaciones en las mujeres inzaeñas: aportes desde Terapia Ocupacional (Tesis de pregrado). Universidad Nacional de Colombia, Bogotá. 
Bundgaard, K. (2005). The meaning of everyday meals in living units for older people. Journal of Occupational Science, 12 (2), 91-101.

Chindoy, B., \& Puenayan, Y. (2009). Las transformaciones de pensamiento y prácticas ocupacionales de las comunidades Kamëntsá y Pastos (Tesis de pregrado). Universidad Nacional de Colombia, Bogotá.

Cutchin, M. P., Aldrich, R. M., Bailliard, A. L., \& Coppola, S. (2008). Action theories for occupational science: The contributions of Dewey and Bourdieu. Journal of Occupational Science, 15(3), 157-165.

Galeano, E., Monsalve, A., \& Wilches, J. (2012). Sentido y significado de las ocupaciones en un grupo de mujeres cuidadoras y no cuidadoras de personas en situación de discapacidad del municipio de Sogamoso. Una experiencia desde Terapia Ocupacional (Tesis de pregrado). Universidad Nacional de Colombia, Bogotá.

Hocking, C. (2009). The challenge of occupation: Describing the things people do. Journal of Occupational Science, 16 (3), 140-150.

Ikiugu, M. (2005). Meaningfulness of occupations as an occupational - life trajectory attractor. Journal of Occupational Science, 12 (2), 102-109.

Leufstadius, C., Erlandsson, L., Björkman T., \& Eklund M. (2008). Meaningfulness in daily occupations among individuals with persistent mental illness. Journal of Occupational Science, 15 (1), 27-35.

Londoño, E. (1988). La conquista del cacicazgo de Bogotá. Boletín Cultural y Bibliográfico, 25 (16). Recuperado de http://web.archive.org/web/20100123163257/http://www. lablaa.org/blaavirtual/publicacionesbanrep/ boletin/boleti5/bol16/conquis.htm

Méndez, J., Bravo, C., Camelo, N. \& Zúñiga, D. (2013). Sentido y significado en las ocupaciones de las mujeres: una experiencia desde el municipio de Inzá, Cauca. Revista Ocupación Humana, 13 (1), 19-30.

Navarrete, E. (2010). Ciencia de la Ocupación: caminos y perspectivas. Revista de Terapia Ocupacional Galicia (A Coruña), 7 (6). 132143.
Persson, D., Erlandsson, L. K., Eklund, M., \& Iwarsson, S. (2001). Value dimensions, meaning, and complexity in human occupation - A tentative structure for analysis. Scandinavian Journal of Occupational Therapy, 8 (1), 7 - 18.

Reed K., Hocking C., \& Smythe, L. (2010). The interconnected meanings of occupation: The call, being-with, possibilities. Journal of Occupational Science, 17 (3), 140-149.

República de Colombia, Ministerio de Agricultura. Decreto 2164 de 1995. Por el cual se reglamenta parcialmente el Capítulo XIV de la Ley 160 de 1994 en lo relacionado con la dotación y titulación de tierras a las comunidades indígenas para la constitución, reestructuración, ampliación y saneamiento de los Resguardos Indígenas en el territorio nacional.

Rowles, G. (2008). Place in occupational science: A life course perspective on the role of environmental context in the quest for meaning. Journal of Occupational Science, 15 (3), 127-135.

Townsend, E. (1997). Occupation: Potential for personal and social transformation. Journal of Occupational Science 4 (1), 18-26.

Trujillo, A., Sanabria, L., Carrizosa, L., \& Parra, E. (2011). Comprensión de la ocupación humana. En C. Rojas, Grupo de Investigación Ocupación y Realización Humana. (Ed.), Ocupación: sentido, realización y libertad. Diálogos ocupacionales en torno al sujeto, la sociedad y el medio ambiente (pp. 27-69). Bogotá: Universidad Nacional de Colombia.

Rojas, C. (Ed.). (2011). Ocupación: sentido, realización y libertad. Diálogos ocupacionales en torno al sujeto, la sociedad y el medio ambiente. Bogotá: Universidad Nacional de Colombia.

Yazdani, F. (2012). The dynamic nature of attributing meaning and value to occupation in Iran. Journal of Occupational Science, 19 (4), 137.

Zemke, R. (2004). Time, Space, and the Kaleidoscopes of Occupation. American Journal of Occupational Therapy, 58 (6), 608-620. 